\title{
Senescent fibroblast-derived Chemerin promotes squamous cell carcinoma migration
}

\author{
Vida Farsam ${ }^{1, *}$, Abhijit Basu ${ }^{1, *}$, Martina Gatzka ${ }^{1}$, Nicolai Treiber ${ }^{1}$, Lars A. Schneider ${ }^{1}$, \\ Medhanie A. Mulaw ${ }^{2}$, Tanja Lucas ${ }^{3}$, Stefan Kochanek ${ }^{3}$, Reinhard Dummer ${ }^{4}$, \\ Mitchell P. Levesque ${ }^{4}$, Meinhard Wlaschek ${ }^{1}$, Karin Scharffetter-Kochanek ${ }^{1}$ \\ ${ }^{1}$ Department of Dermatology and Allergic Diseases, University of Ulm, Germany \\ ${ }^{2}$ Institute of Experimental Cancer Research, University of Ulm, Germany \\ ${ }^{3}$ Department of Gene Therapy, University of UIm, Germany \\ ${ }^{4}$ Department of Dermatology, University Hospital Zurich, Switzerland \\ *These authors contributed equally to this work \\ Correspondence to: Karin Scharffetter-Kochanek, email: karin.scharffetter-kochanek@uniklinik-ulm.de \\ Keywords: cutaneous squamous cell carcinoma (CSCC), senescence-associated secretory phenotype (SASP), tumor migration, \\ Chemerin, chemokine CC-motif receptor-like 2 (CCRL2) \\ Received: May 09, $2016 \quad$ Accepted: October 21, 2016
}

Published: November 18, 2016

\section{ABSTRACT}

Aging is associated with a rising incidence of cutaneous squamous cell carcinoma (CSCC), an aggressive skin cancer with the potential for local invasion and metastasis. Acquisition of a senescence-associated secretory phenotype (SASP) in dermal fibroblasts has been postulated to promote skin cancer progression in elderly individuals. The underlying molecular mechanisms are largely unexplored. We show that Chemerin, a previously unreported SASP factor released from senescent human dermal fibroblasts, promotes CSCC cell migration, a key feature driving tumor progression. Whereas the Chemerin abundance is downregulated in malignant CSCC cells, increased Chemerin transcripts and protein concentrations are detected in replicative senescent fibroblasts in vitro and in the fibroblast of skin sections from old donors, indicating that a Chemerin gradient is built up in the dermis of elderly. Using Transwell ${ }^{\circledR}$ migration assays, we show that Chemerin enhances the chemotaxis of different cSCC cell lines. Notably, the Chemerin receptor CCRL2 is remarkably upregulated in CSCC cell lines and human patient biopsies. Silencing Chemerin in senescent fibroblasts or the CCRL2 and GPR1 receptors in the SCL-1 CSCC cell line abrogates the Chemerin-mediated chemotaxis. Chemerin triggers the MAPK cascade via JNK and ERK1 activation, whereby the inhibition impairs the SASP- or Chemerinmediated cSCC cell migration.

Taken together, we uncover a key role for Chemerin, as a major factor in the secretome of senescent fibroblasts, promoting CSCC cell migration and possibly progression, relaying its signals through CCRL2 and GPR1 receptors with subsequent MAPK activation. These findings might have implications for targeted therapeutic interventions in elderly patients.

\section{INTRODUCTION}

Cutaneous squamous cell carcinoma (cSCC) represents the second most common type of skin cancer worldwide with increasing incidence in elderly individuals [1]. cSCC develops through a multistep process, in which the accumulation of mutations and genetic alterations mainly drive the initiation step [2], while the cellular and molecular alterations of the surrounding microenvironment support the promotion and progression steps [3]. Exposure to ultraviolet (UV) radiation is the main cause for DNA damage and mutations in epidermal keratinocytes as well as in dermal fibroblasts underneath the epidermis [4, 5]. Mutations in the epidermal stem/progenitor cells affecting oncogenes like Ras or tumor suppressor genes such as p53 are 
frequently causal for cSCC initiation [2]. In addition, the DNA damage response pathways in dermal fibroblasts lead to the activation of p53 and p16INK4a and induction of cellular senescence. Senescent fibroblasts have been shown to accumulate over the life span in the skin of rodents, non-human primates and humans [6-8]. Senescent fibroblasts adopt a senescence-associated secretory phenotype (SASP), consisting of inflammatory cytokines, chemokines and matrix remodeling factors that - depending on the biological context - may contribute to tumor suppression or progression [7, 9]. Though the senescent human fibroblasts have been reported to accelerate epidermal tumorigenesis in nude mice, the underlying mediators are largely unknown [10]. It has been proposed that the activation of oncogenic Ras in aged murine skin causes excessive cellular senescence, epidermal stem cell dysfunction, enhanced inflammation, and immune cell skewing towards a T helper cell type 2 , eventually resulting in epidermal dysplasia and cSCC progression [11].

Secreted chemokines are major components of the SASP [12], which play key roles in tumor cell motility, invasion and metastasis [13]. The involvement of chemokine/chemokine receptors in tumor metastasis was first dissected by Müller and colleagues. These authors uncovered the contribution of CXCL12/CXCR4 in breast cancer metastasis [14]. Subsequently, additional evidence substantiated the role of chemokines in tumor progression, particularly in tumor cell migration and invasion $[13,15,16]$. In this context, the CCL21/CCR 7 axis has been proposed to be involved in the development of lymph node metastasis in a variety of different tumors [17-19]; and the CXCL6/CXCR6 axis has been shown to induce the progression of prostate [20] and breast cancers [21]

Of note, we found a remarkable upregulation of C-C chemokine receptor-like 2 (CCRL2) in cSCC primary tumors and cell lines as compared to the normal keratinocytes, their benign counterparts. CCRL 2 has been earlier suggested to contribute to glioblastoma (GBM) cell migration and invasion [22] as well as to colorectal liver metastasis [23]. The known ligand for CCRL2 is the 18-kDa chemoattractant protein Chemerin [24]. Chemerin, also known as tazarotene-induced gene 2 (TIG2) [25] and retinoic acid receptor responder 2 (RARRES2), binds to two other independent receptors termed chemokine-like receptor 1 (CMKLR1 or ChemR23) [26] and G-protein coupled receptor 1 (GPR1) [27]. Overexpression of Chemerin was linked to enhanced tumor angiogenesis and poor clinical outcome in oral squamous cell carcinoma (OSCC) [28], and progression of esophageal squamous cell carcinoma (ESCC) [29, 30]. Increased serum Chemerin level was associated with cellular invasiveness in gastric cancer [31]. Here, we report markedly elevated Chemerin concentrations in the SASP of human senescent dermal fibroblasts, and uncover a previously unreported role for the Chemerin/CCRL2 axis in promoting MAPKdependent cSCC migration.
Our findings may have substantial clinical impact for the development of therapeutic strategies to counteract the SASP-induced progression of cutaneous squamous cell carcinoma in elderly patients.

\section{RESULTS}

\section{The secretome of senescent fibroblasts enhances migration and invasion of cutaneous squamous carcinoma cell lines in vitro}

Enhanced cell motility constitutes an important step in cancer progression and metastasis [32]. As the incidence of cSCCs increases with age and senescent fibroblasts have been suggested to play a causal role in cSCC progression, we investigated the paracrine effects of senescent human dermal fibroblasts (HDFs) on cSCC cell migration and invasion. Conditioned medium (CM) was harvested from senescent and young (early passage) fibroblasts to assess its chemotactic activity on migration of normal human keratinocytes and different cSCC cell lines (SCL-1, SCC13, SCC-12B2 and A431 cells) in a Transwell ${ }^{\circledR}$ chamber migration assay. As depicted in Figure $1 \mathrm{~A}$ and 1B, increased cSCC cell migration was observed in response to senescent CM of FF95 fibroblast strain compared to young CM. Normal keratinocytes did not migrate when exposed to senescent or young fibroblast CM, as opposed to the strong chemotaxis in response to collagen (positive control) [33]. These results were reproduced using CM of all three independent replicative senescent HDF strains (FF95, FFRa and FFPia), confirming that the secretome of all tested senescent HDFs consistently enhanced the migration of SCL-1 tumor cells (Figure 1C). Although to a different extent, a robust migratory response of different cSCC cell lines was induced by CM of senescent fibroblasts of different strains (Figure 1C), these data indicate that the observed enhanced migratory response is similar for all tested cSCC cell lines and senescent fibroblast strains and most likely constitutes a general mechanism of SASP-induced migration. Interestingly, CM of primary stromal fibroblasts derived from cSCC patients at old age displayed similar migratory-promoting effects on SCL-1 cells (Supplementary Figure S1).

Moreover, we observed an increased cell invasion through a reconstituted basement membrane $\left(\right.$ Matrigel $\left.^{\circledR}\right)$ in response to conditioned medium of senescent fibroblasts (FF95 CM) in SCL-1 cancer cells in vitro (Supplementary Figure S2). This phenotype has been previously reported to be mediated through the secretion of active MMP-2 by senescent cancer-associated fibroblasts [34].

\section{The chemoattractant Chemerin is upregulated in senescent fibroblasts}

Earlier we attempted to define the secretome of senescent fibroblasts using an antibody array, mainly confirming the previously published SASP factors 
$[6,35,36]$. Even though these SASP factors, such as CCL5/RANTES [37, 38], were able to significantly stimulate cSCC cell migration (Supplementary Figure S3), they were produced at even higher levels by SCC cells themselves in an autocrine manner, as have been previously reported [39, 40]. Therefore, any significant paracrine contribution from senescent dermal fibroblasts was ruled out.

In a complementary attempt to identify novel SASP factors, we performed PCR array analysis of the chemokine receptors in cSCC cells (Supplementary Figures S4 and S5). Of note, we found a remarkable upregulation of CCRL2 receptor in all tested $\mathrm{CSCC}$ cell lines, a chemokine receptor processing high affinity for Chemerin, the ligand which had not been identified with the conventional screening strategies.

Interestingly, the RARRES2 transcripts encoding the Chemerin protein were increased in all tested senescent fibroblast strains compared to young fibroblasts (Figure 2A). By contrast, with the exception of the A431 cell line, cSCC cells displayed significantly lower RARRES2 mRNA transcripts with a strong downregulation of Chemerin expression as compared to normal cells (keratinocytes) and fibroblasts (Figure 2A).

Enzyme-linked immunosorbent assay (ELISA) revealed increased levels of secreted Chemerin in CM of senescent fibroblasts compared with CM of young fibroblasts with a very low expression in keratinocytes to almost undetectable in cSCC cells (Figure 2B). These data were further confirmed by immunostaining (Figure 2C and 2D), showing significantly higher number of Chemerin-positive fibroblasts $(33.35 \% \pm 8.22$ of total dermal cells) in skin biopsies derived from aged healthy human individuals (age $76 \pm 10$ years, $n=15$ healthy donors) as compared to young humans $(11.82 \% \pm 6.82$ total dermal cells, age $21 \pm 8$ years, $n=13$ healthy donors).

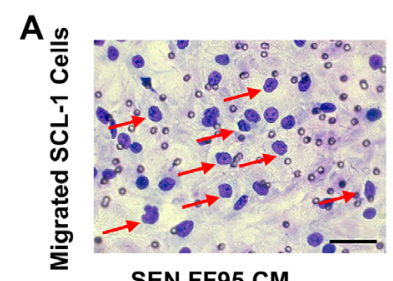

SEN FF95 CM

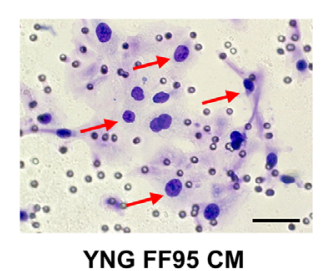

C

口Collagen (Keratinocyte Migration Control)

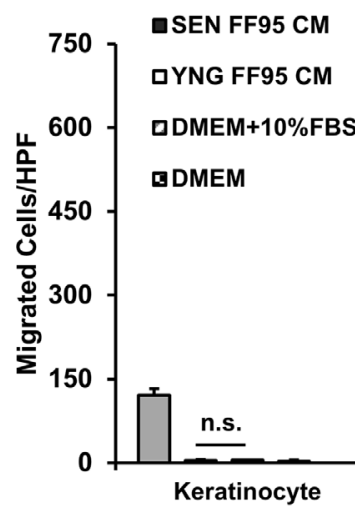

QSEN FF95 CM
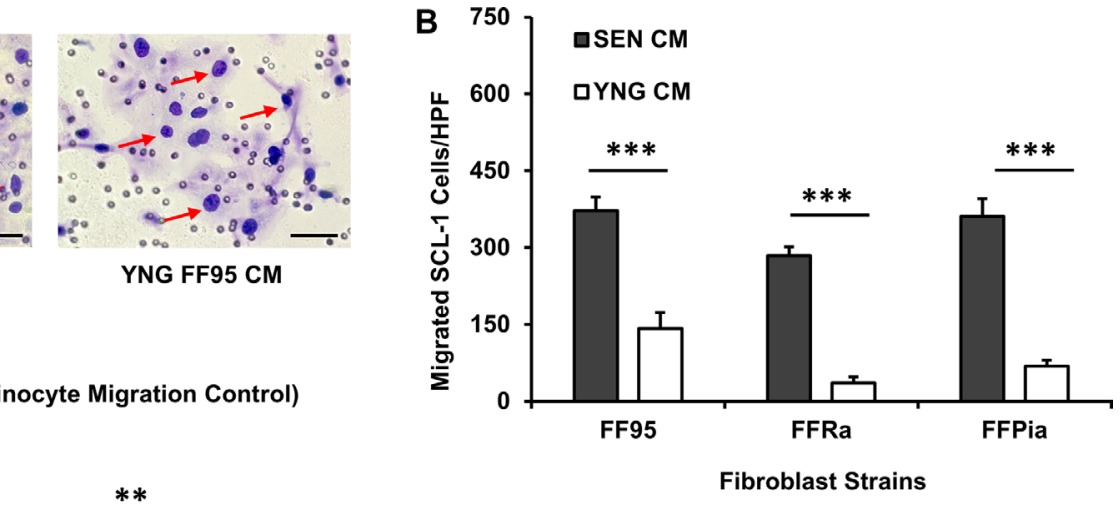

Fibroblast Strains

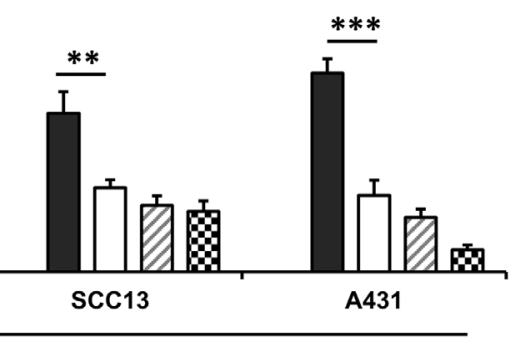

cSCC Cell Lines

Figure 1: Secretome of senescent fibroblasts enhances the migration of squamous cell carcinoma lines. Cell motility was evaluated using the Transwell ${ }^{\circledR}$ chamber migration assay. The lower compartments of the chamber were loaded with conditioned medium $(\mathrm{CM})$ of senescent (SEN) and corresponding young (YNG) fibroblasts and incubated for $14 \mathrm{~h}$ at $37^{\circ} \mathrm{C}$. DMEM containing $10 \%$ fetal bovine serum (FBS) and collagen type I at a concentration of $50 \mu \mathrm{g} / \mathrm{ml}$ served as positive controls. DMEM served as a negative control. Cells stained purple by Diff-Quick staining kit and counted under light microscopy at $\times 100$ magnification (A) Representative images $(\times 200$ magnification, scale bars $=50 \mu \mathrm{m})$ showing migrated SCL-1 cells (pointed with red arrows) at the downside of the membrane in response to CM from senescent vs. young fibroblasts. Quantifications of cell migration proved that (B) Senescent (SEN) conditioned media of human dermal fibroblast strains FF95, FFRa and FFPia enhanced the migration of SCL-1 tumor cells compared with young (YNG) counterparts. Data are shown as mean \pm S.D for $n=3$ replicates. $* * * p<0.001$ calculated by unpaired student $t$-test between SEN CM-treated and YNG CM- treated groups for each fibroblast strains. (C) Conditioned media of FF95 senescent fibroblasts increased the migration of all tested tumor cells (SCL-1, SCC-12B2, SCC-13 and A431) but had no effect on normal keratinocytes. Data are shown as mean \pm S.D for $n=3$ replicates; Graphs represent one of the three independent experiments; ${ }^{*} p<0.05, * * p<0.01$ and $* * * p<0.001$ calculated by unpaired student $t$-test between SEN CM-treated and YNG CM- treated groups for each cell line; HPF $=\times 100$ magnification. (Note that due to low standard deviations of some measurements, error bars are not visible for all data points.). 
Of note, immunostaining of skin biopsies derived from cSCC patients (Supplementary Figure S6) revealed that a large number of Chemerin-releasing FSP $-1^{+}$fibroblasts $(30.83 \% \pm 1.89$ of total stromal cells) were present in the cSCC tumor stroma of older patients (age $80 \pm 5$ years, $n=5$ cSCC patients) as compared to younger cSCC patients (16.04 \pm 3.2 total stromal cells, age $54 \pm 5$ years, $n=5$ cSCC patients).

\section{Chemerin significantly increases the migration of cSCC cell lines in vitro}

To determine whether cSCC cells display a migratory response to the gradients of recombinant human (rh) Chemerin, DMEM containing different concentrations of rh Chemerin was placed in the bottom compartment of the Transwell ${ }^{\circledR}$ chambers with cSCC cell lines seeded on top of the perforated membrane in the upper compartment. Chemerin induced a significant increase in the migration of all assessed cSCC cell lines compared with the untreated control (the number of cells migrating spontaneously in the absence of a chemotactic Chemerin gradient). The increase in the migratory response occurred concentration-dependently, demonstrating a typical bell-shaped response curve with maximal numbers of migrated cells at concentrations between 20 to $40 \mathrm{nM}$ Chemerin (Figure 3A-3D). Based on these results the concentration of $40 \mathrm{nM}$ Chemerin was used for subsequent experiments. Of note, we could show that the migratory response of cSCC cells to rh Chemerin alone occurred
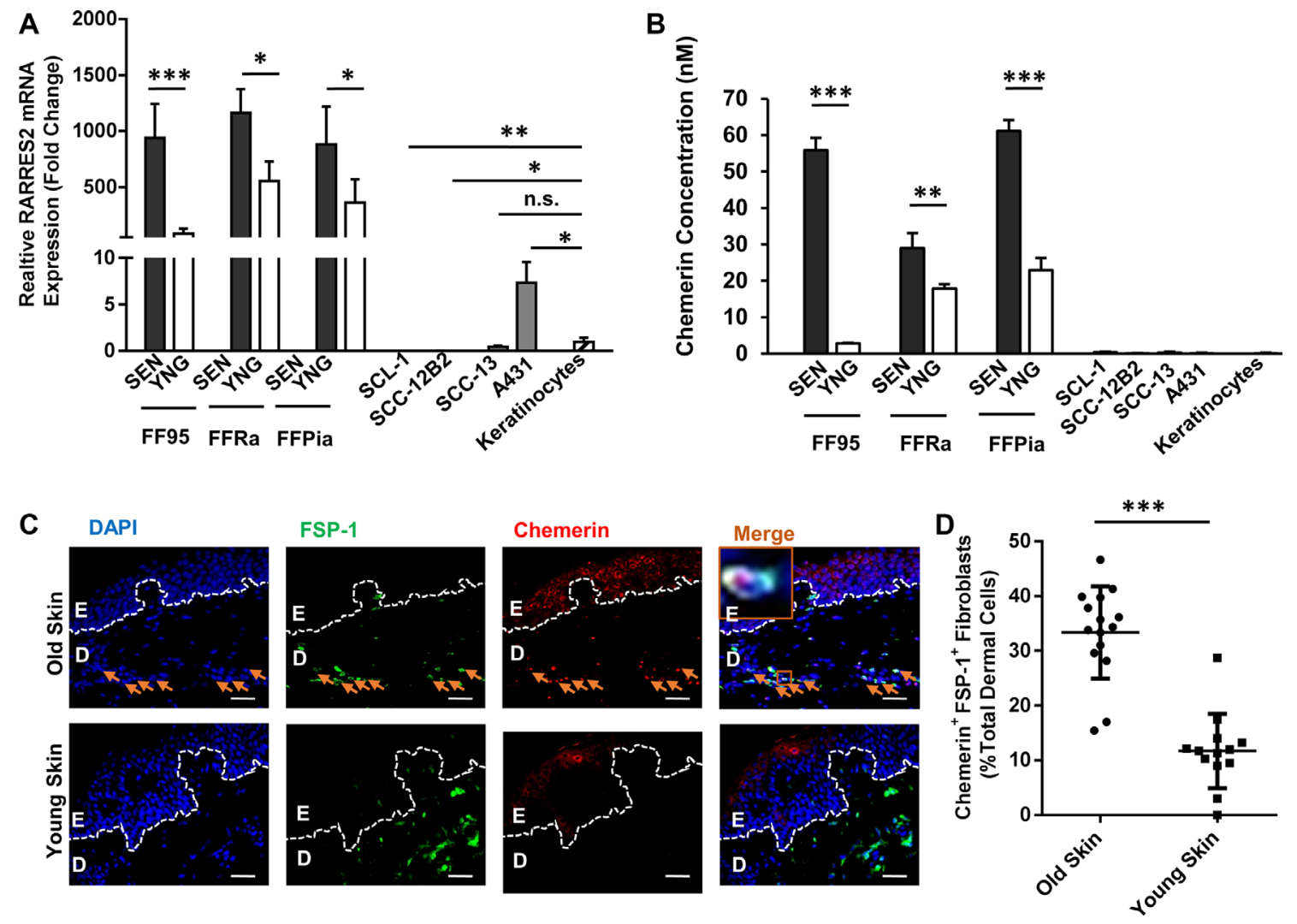

Figure 2: Chemerin is an upregulated SASP factor in human dermal fibroblasts. (A) Graph demonstrating the relative RARRES2 (Chemerin gene) mRNA expression in senescent (SEN) vs. young (YNG) fibroblast of different strains (FF95, FFRa and FFPia) as defined by qRT-PCR. Data are normalized to the expression level of RARRES2 in keratinocytes, confirming that the senescent fibroblasts display the highest, and the cSCC cell lines (SCL-1, SCC12-B2, SCC-13) display the lowest RARRES2 transcripts, respectively. Date are shown as mean \pm S.D for one of three independent experiments of biological replicates $(n=3) ;{ }^{*} p<0.05, * * p<0.01$ and $* * * p<0.001$ calculated by Bonferroni post hoc test after ANOVA. (B) Chemerin secretion was analyzed in the above mentioned cells (normalized to $5 \times 10^{6} \mathrm{cells} / \mathrm{ml}$ ) using ELISA. Data are shown as mean \pm S.E.M for three independent experiments; $* p<0.05, * * p<0.01$ and $* * * p<0.001$ calculated by Bonferroni post hoc test after ANOVA. (Note that due to low standard deviations of some measurements, error bars are not visible for all data points.) (C) Representative photomicrographs of paraffin-embedded human skin sections co-immunostained with antiFSP-1 antibody in green and anti-Chemerin antibody in red, depicting higher abundance of Chemerin in skin dermal fibroblasts of aged (70-year old), compared to young (23-year old) donors. Nuclei were DAPI-counterstained (blue). Appropriate isotype controls were used to determine the background. Scale bars $=50 \mu \mathrm{m}$ at $\times 400$ magnification; Dashed lines delineate epidermis (E) from dermis (D). Orange arrows point to the Chemerin-positive fibroblasts. Orange boxes depict the magnified area. (D) Graph representing the quantification of Chemerinpositive fibroblasts (shown by FSP-1 marker) in the skin dermis of old healthy individuals ( $76 \pm 10$ year, $n=15$ donors) and young ( $21 \pm 8$ year, $n=13$ donors) calculated from minimum 5 technical replicates. ${ }^{* * *} p<0.001$ by two-tailed student $t$-test. 
at a physiological Chemerin concentration of 20-40 nM ( $<150$ cells/HPF), while the CM of senescent fibroblasts induced an even stronger migratory response of cSCC cells $(>300$ cells/HPF), suggesting that other SASP factors in addition to Chemerin are contributing. In fact, we identified the SASP factor RANTES/CCL5 to induce cSCC cell migration (Supplementary Figure S3).

Of note, Chemerin does not induce cSCC cell proliferation in vitro as shown by MTT test and BrdU incorporation assays (Supplementary Figures S7A-S7E). These observations provide convincing evidence in favor of the hypothesis that migration and proliferation are mutually exclusive in cancer cells $[41,42]$.

\section{Chemerin depletion diminishes the potential of senescent fibroblast-derived SASP to induce cSCC cell migration}

To further assess whether Chemerin contributes to the SASP-induced migratory response of cSCC cells, Chemerin was depleted from senescent fibroblasts using Chemerin-specific silencing RNAs. Two days after transfection, RARRES2 transcripts and Chemerin secretion dramatically decreased to less than $5 \%$ of the mock-treated fibroblasts as shown with qRT-PCR and ELISA (Figure 3E and 3F), respectively. Of note, CM derived from Chemerin-silenced senescent fibroblasts showed a significant reduction (over 30\%) in stimulating the migratory response of SCL-1 cells (Figure $3 \mathrm{G}$ ). This finding was confirmed with four independent silencing RNAs, indicating that Chemerin is one of the major SAPS factors of senescent dermal fibroblasts, which induces cSCC cell migration.

\section{The Chemerin receptor CCRL2 is upregulated in cSCC cells}

As mentioned above, the higher level of CCRL2 receptor expression was detected on cSCC cells compared with normal cells using the PCR array analysis.

We further employed qRT-PCR, Western blot and flow cytometry analysis to evaluate the expression of CCRL2 and other Chemerin receptors including CMKLR1 and GPR1 in cSCC cell lines and normal keratinocytes (Figure 4). Notably, CCRL2 mRNA was significantly increased in all cSCC cells ( $>25$-fold) compared to normal keratinocytes (Figure 6A, 6B and Supplementary Figures S4 and S5). GPR1 mRNA was also detectable in cSCC cell lines, although the transcripts were substantially less abundant in comparison with normal keratinocytes (Figure 4A and 4C).

Transcripts of CMKLR1 were neither detected in cSCC cell lines, nor in keratinocytes (Figure 4A).

Using flow cytometry analysis, the cell surface expression of CCRL2, GPR1 and CMKLR1 was analyzed, revealing high expression levels of CCRL2 $(77.7 \% \pm 4.6)$ and low expression of GPR1 $(3.55 \% \pm 0.87)$ on SCL-1 cells in comparison to a significantly lower level of CCRL2 (8.7\% \pm 1.1$)$ and higher GPR-1 (13.1\% \pm 7.92) on keratinocytes (Figure 4D and 4E). Neither SCL-1 cells nor keratinocytes expressed CMKLR1 at detectable levels (data not shown).

Western blot analysis confirmed the upregulation of CCRL2 at the protein level; Consistent with the FACS analysis and qRT-PCR data, SCL-1, SCC-12B2, SCC-13 and A431 strongly expressed CCRL2 compared with normal keratinocytes (Figure 4F).

To confirm the clinical significance of CCRL2 expression, primary tumors from cSCC patients $(n=8)$ were subjected to immunofluorescence staining. CCRL2 staining was mainly observed in the invading edges of cSCC cells. By contrast, in normal skin from healthy individuals, epidermal keratinocytes displayed no or very low CCRL2 expression (Figure 4G).

Hence, CCRL2 constitutes the most abundant Chemerin receptor in cSCC cells, suggesting that it may favor the enhanced migration of $\mathrm{cSCC}$ tumor cells to invade the Chemerin-rich dermis.

\section{Role of CCRL2 and GPR1 in Chemerin-mediated cSCC cell migration}

Enhanced CCRL2 expression on cSCC cells indicates that this receptor might play a role in the migratory response to Chemerin. We therefore assessed whether the knockdown of CCRL2 expression using lentivirus-based short hairpin RNAs (shRNAs) could suppress the cSCC cell migration. Likewise, the GPR1 receptor was also lentivirally-silenced from SCL-1 cells. Successful transcriptional silencing of GPR1 and CCRL2 $(>50 \%)$ in sorted populations of mCherry-positive (GPR1) or eGFP-positive (CCRL2) SCL-1 cells (Figure 5A-5B) was verified using qRT-PCR (Figure 5C-5D).

The GPR1-silenced and CCRL2-silenced cells were subjected to Transwell ${ }^{\circledR}$ chamber migration assays. Relative cell migration (Chemerin/ ${ }_{\text {Untreated }}$ ) was calculated in each group as the ratio of cells migrated towards a Chemerin gradient $(40 \mathrm{nM})$ to the cells migrated randomly in the negative control (untreated cells in serum-free DMEM). The results showed that the directed cell migration in response to rh Chemerin was reduced to a similar level as random migration in GPR1-knockdown or CCRL2-knockdown cells (Chemerin/ ${ }_{\text {Untreated }} \sim 1$ ) as compared to the scrambled-transfected cells (Chemerin/ Untreated $>2$ ). In other words, the shRNAmediated silencing of either CCRL2 or GPR1 blocked the Chemerin-induced migration by $>50 \%$ in SCL-1 cells in comparison to scrambled control (Figure 5E). Consistent results were achieved with both independent shRNAs targeting CCRL2 and GPR1 (Figure 5E).

These results support the notion that CCRL2 and GPR1 constitute the responsible receptors for Chemerininduced cSCC cell migration. This is consistent with our 
observation that keratinocytes, despite expressing high levels of GPR1, are not responsive to Chemerin, probably due to the very low CCRL2 expression (data not shown).

\section{Activation of MAPK signaling pathway in response to Chemerin}

To further elucidate the molecular mechanism underlying Chemerin-induced cSCC cell migration, we explored signaling pathways downstream of the Chemerin receptors CCRL2 and GPR1, both belonging to the family of G-protein coupled receptors (GPCRs). We employed a GPCR Cignal ${ }^{\circledR}$ Finder Array, and transfected SCL-1 cells with luciferase reporter DNA constructs containing transcription factor elements (TFEs), and assessed the reporter activities in response to rh Chemerin stimulation. $\mathrm{Rh}$ Chemerin enhanced Mitogen-activated protein kinase (MAPK) activities of both c-Jun N-terminal kinases (JNK) and extracellular-signal-regulated kinase (ERK) signaling pathways in SCL-1 cells after an incubation period of $1 \mathrm{~h}$ (Figure 6A). The activation of JNK and ERK persisted for up to 24 hours (data not shown).

Activation of MAPK pathways in response to rh Chemerin was further confirmed at the protein level by Western blot analysis. Increased phosphorylated forms of ATF2, SEK1, c-Jun and ERK1/2, were detected in rh Chemerin-treated SCL-1 cells compared with untreated cells (Figure 6B).

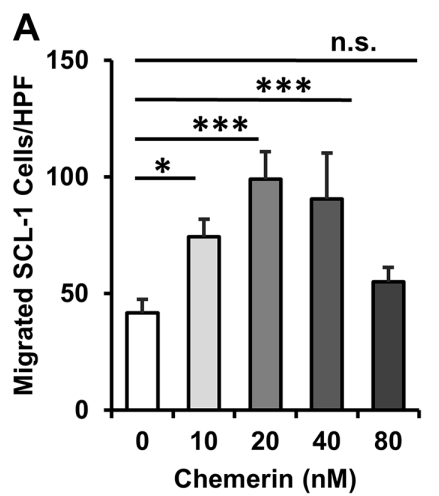

E

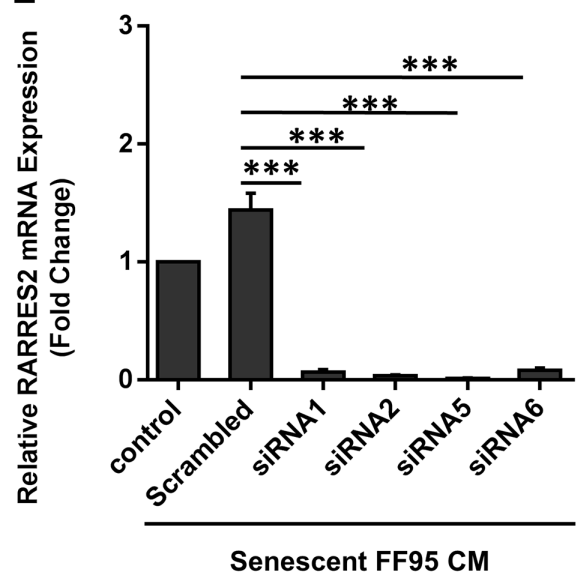

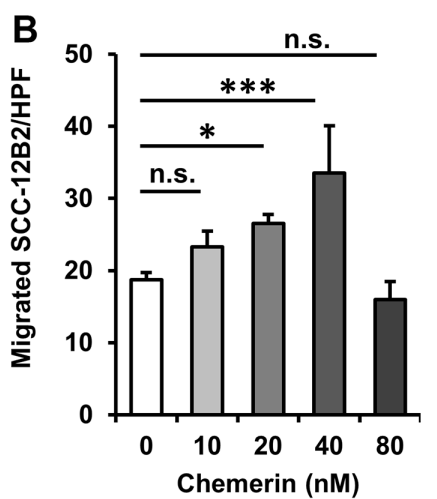

$\mathbf{F}$

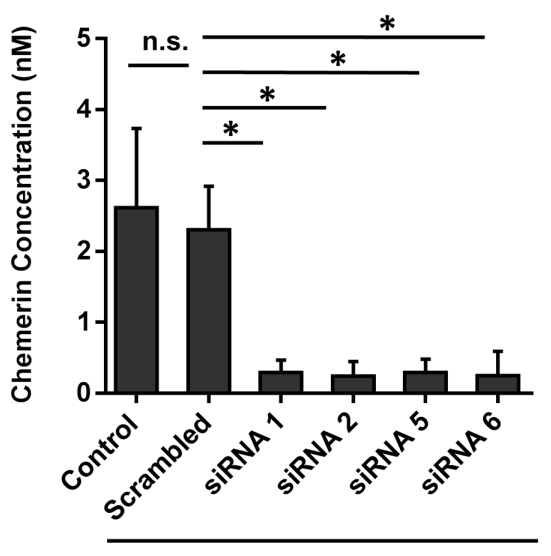

Senescent FF95 CM

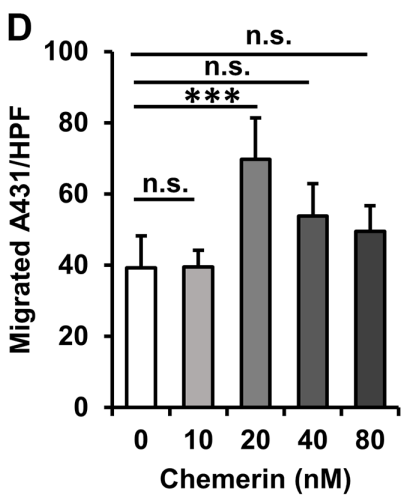

G

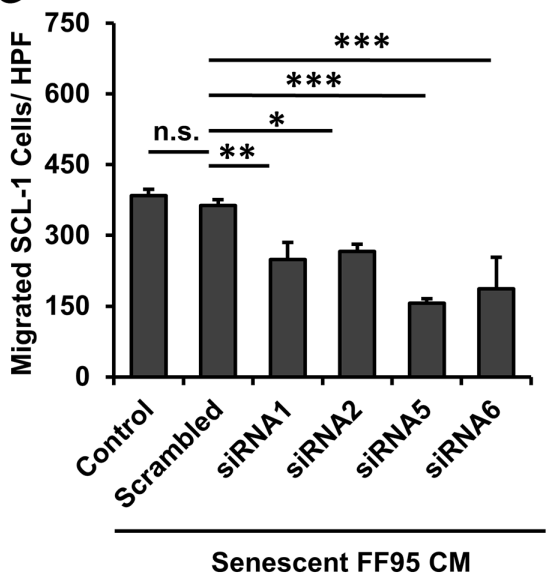

Figure 3: Chemerin stimulates migration of squamous carcinoma cell lines. Graphs demonstrating the bell-shape curves of chemotactic response to the increased concentrations of Chemerin in (A) SCL-1, (B) SCC-12B2, (C) SCC-13 and (D) A431 cells using the Transwell ${ }^{\circledR}$ chamber migration assay. Date are shown as mean \pm S.D for one of three independent experiments with $n=4$ replicate wells; n.s. $=$ non-significant, ${ }^{*} p<0.05, * * p<0.01$ and $* * * p<0.001$ in comparison to random migration control with no Chemerin treatment calculated by Bonferroni post hoc test after ANOVA. The role of Chemerin in mediating cSCC cell migration was confirmed by silencing the Chemerin gene (RARRES2) in senescent fibroblasts and assessing their ability to induce SCL-1 cell migration (E) Transcript level of RARRES2 was quantified by qRT-PCR in senescent fibroblasts $42 \mathrm{~h}$ post treatment with siRNAs, confirming the successful gene silencing compared to scrambled control. Data are shown as mean \pm S.E.M from three independent experiments. ${ }^{* * *} p<0.001$ calculated by Bonferroni post hoc test after ANOVA. (F) Chemerin protein level (normalized to $5 \times 10^{6}$ cells $/ \mathrm{ml}$ ) was measured 48 hours after treatment with siRNAs or scrambled control. Data are shown as mean \pm S.E.M from four independent experiments; $*^{*}<0.05$ calculated by Bonferroni post hoc test after ANOVA. (G) Conditioned media derived from Chemerin-silenced fibroblasts (siRNA 1, 2, 5 and 6 ) were used to induce the migration of SCL-1 cells using the Transwell ${ }^{\circledR}$ chamber migration assay. Conditioned media derived from mock-treated fibroblasts (Scrambled) and senescent fibroblasts with no treatment (control) were used as controls. Shown is one representative of four independent experiments, each with triplicate samples. ${ }^{*} p<0.05,{ }^{* *} p<0.01$ and ${ }^{* * *} p<0.001$ calculated by Bonferroni post hoc test after ANOVA. 
A

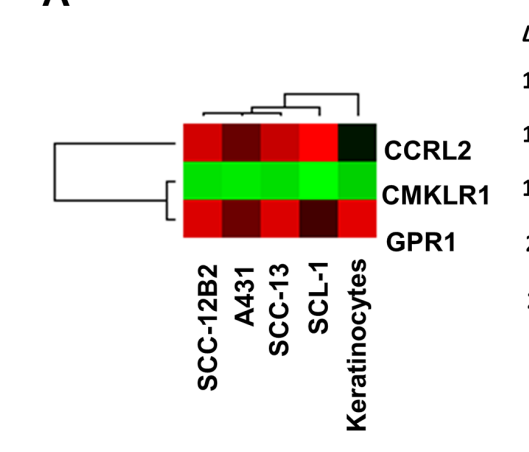

D
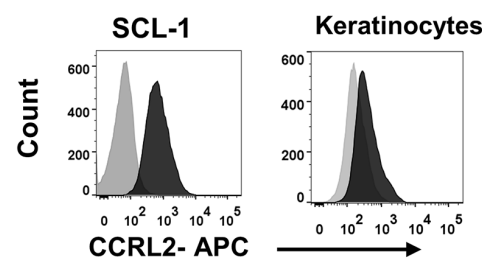

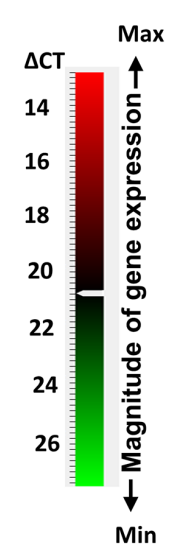

. $\mathrm{B}$

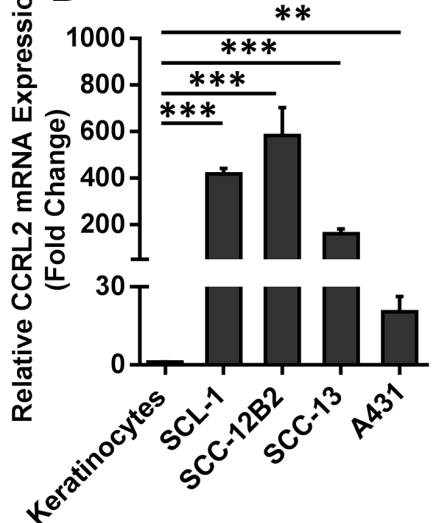

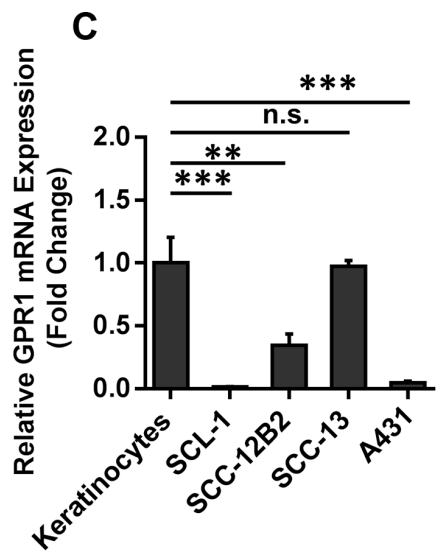
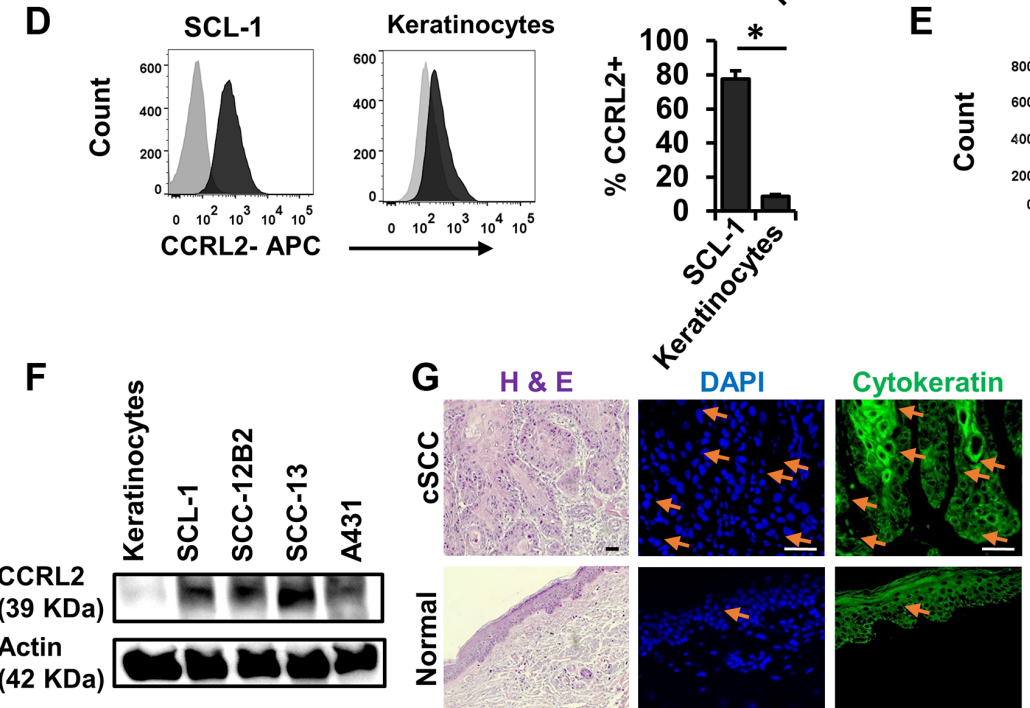

E
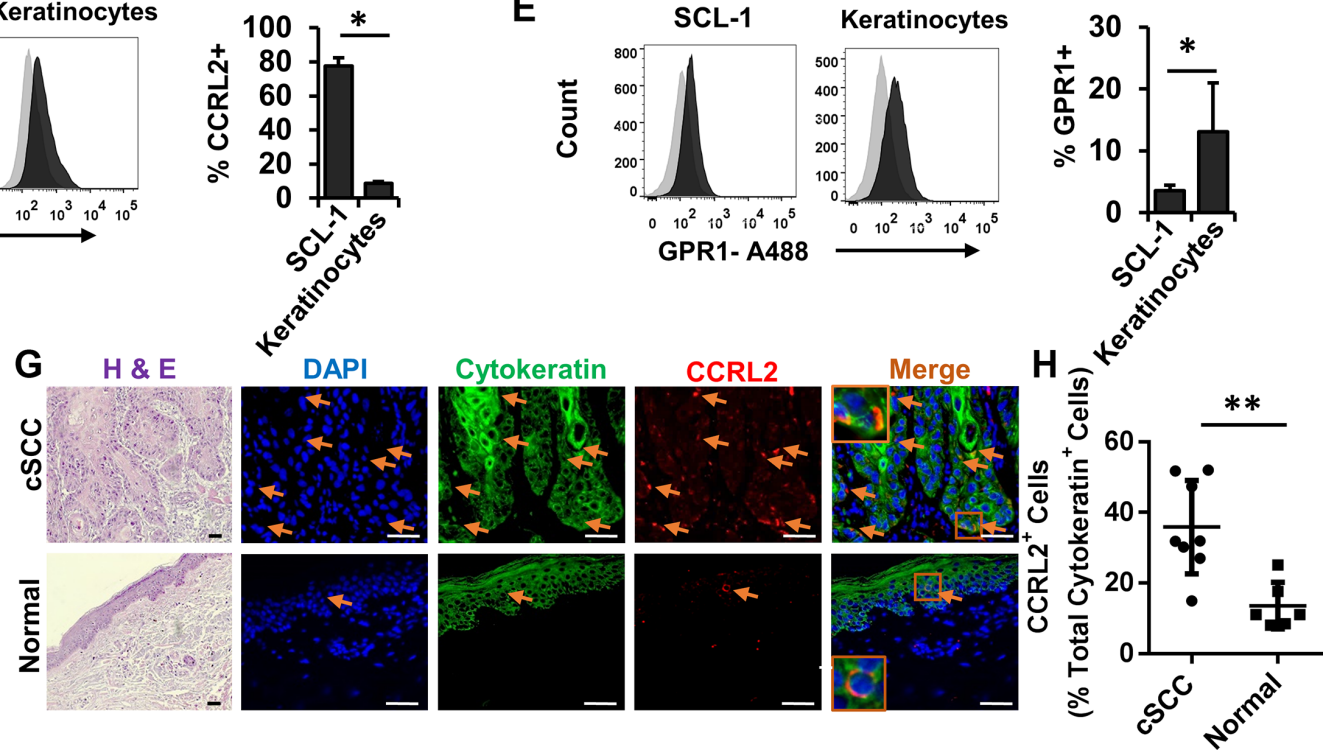

Figure 4: CCRL2 is the upregulated Chemerin receptor in cutaneous carcinoma cell lines and human squamous cell carcinoma. (A) Heat map representing the gene expression profile of Chemerin receptors CCRL2, GPR1 and CMKLR1. Relative gene expression was calculated from all three independent experiments normalized to the level of Actin housekeeping gene expression, and relative to the mean expression in normal keratinocytes. Genes were rank-ordered by Pearson correlation and depicted by a pseudo color scale based on qRT-PCR delta-CT values with green and red demonstrating low and high mRNA abundance, respectively. CMKLR1 had very low to no mRNA transcripts; CCRL2 was highly upregulated in CSCC cells; and GPR1 was either downregulated or not changed in cSCC cells compared to keratinocytes. (B-C) Graphs depict the relative fold changes of CCRL2 and GPR1 mRNA expression in cSCC lines compared with normal keratinocytes as defined by qRT-PCR. Shown is one representative of three independent experiments, each with triplicate samples. ${ }^{*} p<0.05,{ }^{* *} p<0.01$ and ${ }^{* * *} p<0.001$ calculated by Bonferroni post hoc test after ANOVA. (Note that due to low standard deviations of some measurements, error bars are not visible for all data points.) (D-E) Cell surface expressions of CCRL2, GPR1 and CMKLR1 were assessed by flow cytometry analysis in SCL-1 cells and normal keratinocytes. Displayed are the representative fluorescence histograms depicting the relative fluorescence intensity of cells stained with (D) APC-conjugated anti-CCRL2 and (E) A488conjugated anti-GPR1 antibodies (black histograms) compared with isotype control antibodies conjugated with corresponding fluorochromes (gray histograms). The expression of CMKLR1 was not detectable (not shown). Bar charts represent one of three independent experiments demonstrating the mean percentage \pm S.D. for (d) CCRL2-positive and (E) GPR1-positive SCL-1 cells vs. keratinocytes. ${ }^{*} p<0.05$, ${ }^{* *} p<0.01$ and ${ }^{* * *} p<0.001$ calculated by two-tailed student $t$-test $(n=8$ biological replicates). (F) Representative Western bot analysis of cSCC and keratinocyte cell lysates, confirming the elevated protein level of CCRL2 in cSCC cells compared with normal keratinocytes. (G) Representative photomicrographs of skin biopsies derived from patients suffering from invasive $\mathrm{CSCC}$ vs. normal healthy controls stained for Hematoxylin and Eosin (H \& E), CCRL2 in red and cytokeratin in green. Nuclear was stained with DAPI (blue). Arrows show the CCRL2-positive cells. Appropriate isotype controls were used to determine the background. H\&E images were taken at $\times 200$ magnification and immunofluorescence at $\times 400$ magnification; Scale bars $=50 \mu \mathrm{m}$. Orange boxes depict the magnified area. (H) Graph representing the quantification of CCRL2-positive epidermal-derived cells in the skin specimens of patients suffering from $\operatorname{cSCC}(n=8)$ vs. normal healthy individuals $(n=6) . * *<0.01$ by two-tailed student $t$-test. 


\section{Inhibition of MAPK pathway abrogates cSCC cell migration}

Next we addressed the question whether the inhibition of the MAPK pathway can suppress Chemerinor SASP-mediated migration of cSCC tumor cells. Notably, the ERK1/2 inhibitor FR180204 [43], and the JNK inhibitor SP600125 [44], in a concentrationdependent manner, mitigated the ability of senescent $\mathrm{CM}$ to induce SCL-1 cell migration. The SASP-mediated SCL-1 cell migration was almost completely abolished following treatment with $30 \mu \mathrm{M}$ FR180204 and to a lesser extent with SP600125 (Figure 6C). BIRB796, a specific p38 inhibitor [45, 46], did not significantly decrease the SASP-induced migration of SCL-1 cells.
A concentration of $10 \mu \mathrm{M}$ FR180204 or SP600125 but not BIRB796 blocked the rh Chemerin-induced SCL-1 cell migration (> 70\%) in comparison to control (Figure 6D), suggesting that Chemerin regulates the cSCC cell migration through ERK1/2 and JNK- but independently of the p38 MAPK pathway.

The used concentrations were non-toxic as shown by MTT assays (Supplementary Figure S7F).

\section{DISCUSSION}

Accumulating evidence suggests that the senescence-associated secretory phenotype (SASP) is one of the major causal links between the age-associated alterations in tissue microenvironment and the rising

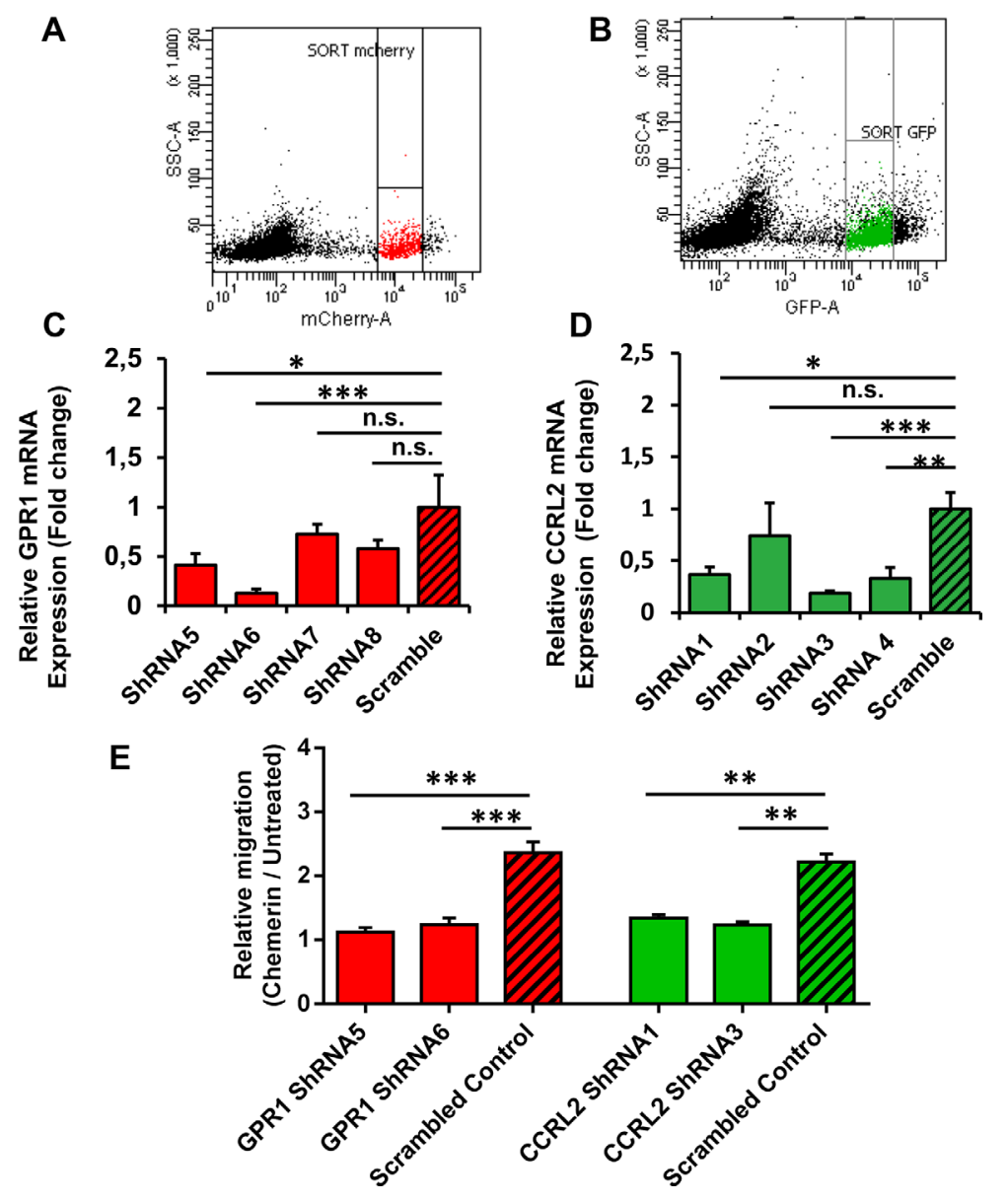

Figure 5: CCRL2 and GPR1 receptors are required for Chemerin-stimulated squamous cell carcinoma migration. In order to study the role of Chemerin receptors in mediating CSCC cell migration, SCL-1 cells were transduced with lentivirus encoding shRNAs against GPR1 and CCRL2. The GPR1 shRNA-expressing lentiviral vector and the CCRL2 shRNA-expressing lentiviral vector contained mCherry and eGFP proteins, respectively, which enabled us to isolate positively-transduced cells using fluorescence-activated cell sorting (FACS). (A-B) Representative FACS dot plots showing the final gating strategies to sort mCherry- and eGFP-expressing cells. (C-D) The efficiency of CCRL2 and GPR1 knockdown in SCL-1 cells was validated by qRT-PCR. Data are presented as bar charts, representing the mean expression fold changes relative to scrambled controls \pm S.D. ${ }^{*} p<0.05,{ }^{* *} p<0.01$ and ${ }^{* * *} p<0.001$ calculated by ANOVA $(n=3)$. (E) Graph indicating that CCRL2 and GPR1 silencing suppresses Chemerin-mediated SCL-1 cell migration. The relative migration (Chemerin/ Untreated $)$ was calculated in each group as the ratio of cells migrated towards the Chemerin gradient $(40 \mathrm{nM})$ to the cells migrated randomly in the negative control (serum-free DMEM). Data are presented as Mean \pm S.E.M. for 3 independent experiments; ${ }^{*} p<0.05,{ }^{* *} p<0.01$ and ${ }^{* * *} p<0.001$ calculated by Bonferroni post hoc test after ANOVA. 
incidence of cancer $[9,11,47]$. It has been recently shown that the SASP from senescent stromal fibroblasts inhibits the RhoA/ROCK/myosin-dependent cell contractility leading to aggressive cell motility in human breast cancer cells [48]. However, the exact key SASP factors and the mechanisms involved in cSCC cell migration have not been fully elucidated. Here we showed that Chemerin, released by senescent dermal fibroblasts, exerts a strong chemotactic activity in cSCC cells and thus contributes to enhanced cSCC cell migration via activation of MAPK

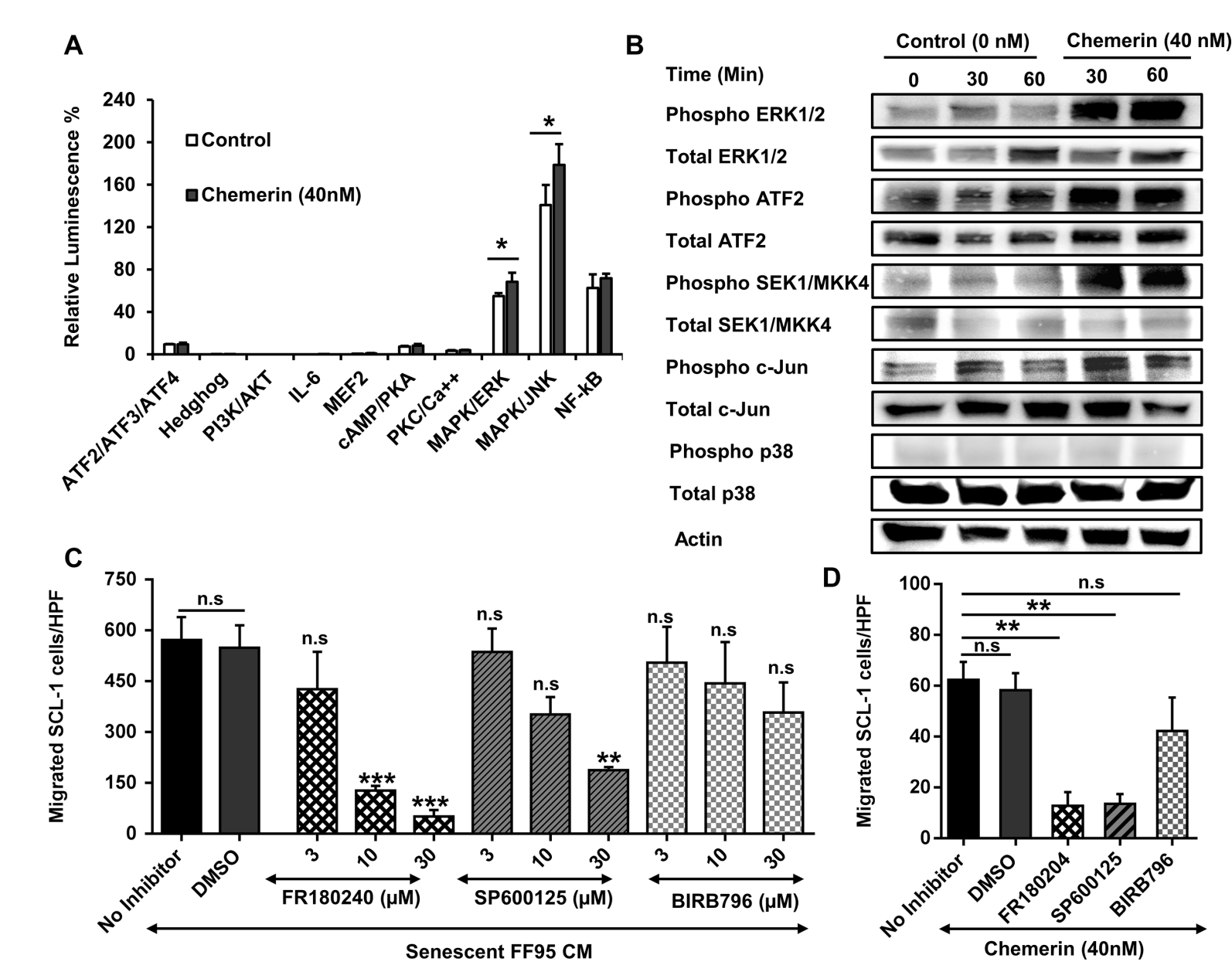

pathway. These findings were corroborated by our observation that the putative Chemerin receptor, CCRL2, was remarkably upregulated in cSCC tumor cells, which, together with the GPR1 receptor, mediated the response to Chemerin.

In general, Chemerin is known to be involved in regulating adipogenesis and lipid metabolism [49], inflammation and leukocyte trafficking [50], and endothelial angiogenesis and MMP production [51]. Dysregulated expression of Chemerin has been correlated

Figure 6: Role of MAPK signaling pathway in the regulation of Chemerin-mediated cell migration. (A) In order to define the signaling pathway activated by Chemerin, we used a G-protein couple receptor (GPCR) Cignal Finder ${ }^{\circledR}$ Reporter Array consisting of inducible transcription factor response constructs encoding the firefly luciferase reporter gene. SCL-1 cells were transfected with each reporter constructs and further treated with DMEM containing $40 \mathrm{nM}$ rh Chemerin or no Chemerin (control) for 1 hour. The dual-luciferase assay was developed and the results were expressed as the percentage of relative luminescence signal according to the manufacturer's instructions. Results highlighted that Chemerin activates JNK and ERK1/2 MAPK signaling pathway. Shown is a representative of three independent experiments presented as Mean $\pm \operatorname{S.D}(n=3) .{ }^{*} p<0.05$ calculated by unpaired student $t$-test. (Note that due to low standard deviations of some measurements, error bars are not visible for all data points.) (B) To confirm the Cignal Finder ${ }^{\circledR}$ Reporter Array data, SCL-1 cells were cultured in the presence and absence of $40 \mathrm{nM}$ rh Chemerin for 30 and 60 minutes and protein lysates were analyzed by Western blot for phosphorylated and total amounts of MAPK key proteins. (C) We investigated whether inhibition of MAPK pathway dampens the migration of SCL-1 cells stimulated by Chemerin-rich conditioned media of senescent fibroblasts. The graph represents the concentration-dependent inhibition of SCL-1 cell migration in response to senescent fibroblast CM by SP600125 (JNK inhibitor), FR180204 (ERK1/2 inhibitor) and BIRB796 (P38 inhibitor). DMSO served as a vehicle control. Data are presented as Mean \pm S.E.M. for 3 independent experiments; ${ }^{*} p<0.05, * * p<0.01$ and $* * * p<0.001$ in comparison to DMSO control calculated by Bonferroni post hoc test after ANOVA; HPF $=\times 100$ magnification $(\mathbf{D})$ Bar graph showing the effect of MAPK inhibitors $(10 \mu \mathrm{M})$ on Chemerin-mediated SCL-1 cell migration. Data are presented as mean \pm S.E.M. for 3 independent experiments; ${ }^{* * *} p<0.001$ calculated by Bonferroni post hoc test after ANOVA. $\mathrm{HPF}=\times 100$ magnification. 
with tumor progression in glioma [52], squamous cell carcinoma of oral tongue [28], esophageal cancer [30] and with tumor suppression in melanoma [53]. However, the biological effects of differential expression of Chemerin in tumor and stromal cells in the context of tumor migration have not been sufficiently explored. In the present study, based on the following findings, we conclude that the Chemerin/CCRL2/GPR1 axis is induced by the paracrine action of senescent fibroblasts, causing enhanced cSCC migration and most likely progression.

First, a comprehensive transcriptional analysis of the chemokine receptor expression in cSCC cell lines revealed a remarkable upregulation of CCRL2 in cSCC cell lines compared to normal keratinocytes (Supplementary Figures S4 and S5), a finding which was further confirmed with the immunostaining of patient tumor biopsies. The elevated CCRL2 expression in other tumors including malignant breast cancer [54], high grade glioblastoma [22] and cervical carcinoma [55] has been previously postulated to be associated with cell migration, invasion and poor prognosis, irrespective of whether its biological ligands were present or not. Thus, the high abundance of CCRL2 receptor on cSCC cell surface implicates a crucial role in tumor progression. We assumed that the CCRL2 upregulation confers a migratory advantage for the epidermal cSCC tumor cells, particularly at the invasive front of the epidermis-dermis junction, facilitating their migration towards the dermis, where a gradient of its high-affinity ligand, Chemerin, is established by senescent fibroblasts. Consistent with this notion, we detected significantly high Chemerin concentrations in senescent dermal fibroblasts in vitro, as well as in the dermal fibroblasts of old healthy individuals and in the stromal fibroblasts of old cSCC patients in situ. This expression pattern was significantly different in the young healthy skin, exhibiting lower Chemerin abundance in the dermis vs. epidermis, as previously demonstrated by Banas et al. [56]. Interestingly, in line with a previous publication [57] we found that Chemerin production was significantly decreased in cSCC cells compared to normal keratinocytes.

Second, as to the functional consequences of senescence-associated Chemerin upregulation in old skin, we showed that rh Chemerin increased the directed cSCC cell migration; and silencing of Chemerin in senescent fibroblasts significantly reduced their potential to induce cSCC cell migration. To our knowledge, this is the first report on the role of Chemerin in cSCC cell migration, although Chemerin has been previously reported to promote gastric cancer and esophageal cancer cell invasion via induction of VEGF, MMPs and IL-6 [30, 31].

Third, we showed that, in the absence of CMKLR1, both the CCRL2 and GPR1 receptors are required for Chemerin-mediated cSCC cell migration, as silencing of either receptors suppressed the migratory response of cSCC cells to a Chemerin gradient. In agreement with this finding, previous reports have demonstrated that the CCRL2 upregulation through an undefined ligand contributes to glioblastoma cell migration [22] and colorectal metastasis [23]. By contrast, CCRL2 overexpression was reported to inhibit CCL2-induced phosphorylation of p38 MAPK leading to decreased chemotaxis and invasion of human breast cancer cells [58]. Thus, the involvement of CCRL2 in Chemerininduced cell migration, as a key step in tumor progression, is still confounding as to different tumor entities. This notion is based on the finding that CCRL2 possesses an altered amino acid sequence (QRYLVFL in huCCRL2 and QRYRVSF in mCCRL2 instead of the conserved DRYLAIV motif) in the second intracellular loop which is essential for signal transduction [24]. In this context, our data on GPR1/CCRL2/Chemerin-induced cell migration are novel and would fit to the current model of CCRL2 action, proposed by Zabel and colleagues [24]. The model suggests that Chemerin binds to CCRL2 receptors via its amino-terminal domain, which in consequence increases the abundance of cell surface-bound Chemerin and its local concentration. Chemerin then, in a juxtacrine fashion, reacts with other Chemerin receptors (e.g. CMKLR1 or GPR1) via its free carboxyl-terminal residues. More detailed analysis would, however, be required to further delineate the exact mechanism whereby Chemerin interacts with GPR1 and/or CCRL2 to stimulate cSCC migration.

Fourth, we showed that Chemerin triggers the mitogen-activated protein kinase (MAPK) signaling pathway in CCRL2 ${ }^{\text {high }}$ GPR $1^{\text {low }}$ SCL-1 cells which express no detectable CMKLR1 proteins. Previously, Chemerin was shown to activate the p38 and ERK1/2 MAPK pathways in gastric cancer cells [31], and PI3K, Akt, and p38 in CMKLR1-expressing macrophages [59]. In the current study, using the GPCR Cignal ${ }^{\circledR}$ Finder array and Western blot analysis, the ERK1/2 and JNK emerged as the key downstream mediators of Chemerin-induced signaling in cSCC cells. This finding indicates that Chemerin, depending on the cell type and the receptor expression can activate different subtypes of MAPK pathway. The involvement of both ERK $1 / 2$ and JNK in cell migration and invasion is not unexpected and, in fact, has been wellestablished in various cancers [60]. We observed that the inhibition of ERK $1 / 2$ and JNK with small molecule inhibitors abolished the migration-promoting potential of Chemerin and senescent fibroblast CM in cSCC cells. This implies that majorly Chemerin, and possibly other SASP factors, reinforce MAPK as a common signaling pathway to induce tumor-cell migration. This is a clinically relevant finding indicating that the inhibition of the MAPK pathway may prevent cSCC migration and metastasis.

Apart from Chemerin, other SASP factors among them RANTES/CCL5 contribute to cSSC cell migration. The role of several SASP factors in tumor cell migration has previously been investigated. For instance, the two 
well-known SASP factors, CXCL8/IL-8 and CCL2/ MCP-1, increase the migration of colorectal cancer cells [61]. Also, production of a Wnt antagonist named sFRP2 released from senescent dermal fibroblasts has been reported to decrease $\beta$-catenin and microphthalmiaassociated transcription factor (MITF). This in conjunction with the loss of the redox effector protein APE1, a multifunctional protein with both DNA repair and transcriptional regulatory activities, eventually contributes to tumor progression and metastasis in melanoma [62].

In summary, we here uncovered the previously unreported GPR1/CCRL2/Chemerin axis in cSCC tumor cells which - via the interrelated activation of the ERK1/2 and JNK- promotes directed migration and most likely tumor progression. Given the current demographic development, this is particularly relevant as Chemerin is up-regulated in senescent fibroblasts in the skin of elderly individuals.

Our findings with the identification of target molecules responsible for skin cancer cell migration/ invasion hold substantial promise for the development of novel treatment strategies to overcome the deleterious effects of SASP in elderly patients.

\section{MATERIALS AND METHODS}

\section{Cell lines and primary cells}

Human cutaneous squamous cell carcinoma (cSCC) lines SCC-12B2 and SCC-13 [63] were kindly provided by Dr. J.G. Rheinwald (Harvard Medical School, Boston, MA). Human SCC line A431 was purchased from the American Type Culture Collection (ATCC, Manassas, VA, USA).

Human SCL-1 cell line [64] was a gift from Dr. P. Boukamp (DKFZ, Heidelberg, Germany). Primary human dermal fibroblast (HDF) strains FF95, FFRa and FFPia, and primary normal human epidermal keratinocytes (NHEKs) were established from the foreskin of healthy males having undergone circumcision according to the method described earlier $[65,66]$. Primary cSCCassociated stromal fibroblasts were derived from surplus material removed from patient biopsies (F-SCC3, 88 year-old male and F-SCC1160524, 92 year-old male) at the University Hospital Zurich, funded by the University Research Priority Program (URPP). All patients included in this study have signed a patient release form, which has been approved by an ethics committee and assigned the numbers EK647, and EK800.

\section{Cell culture}

SCL-1, A431, HDFs and cSCC-associated primary stromal fibroblasts (F-SCC3 and F-SCC1160524) were maintained in Dulbecco's Modified Eagle's Medium (DMEM; GIBCO-BRL, Germany) supplemented with $10 \%$ fetal bovine serum (FBS; Biochrom, Berlin,
Germany), 2 mM L-glutamine (Biochrom), 100 U/ml streptomycin and penicillin (Biochrom), which will be referred to as "DMEM growth media" throughout the manuscript.

NHEKs, SCC12-B2 and SCC13 cells were cultured in complete Keratinocyte Growth Medium M2 (\#20011; Promocell, Heidelberg, Germany). Collagen-coated $\left(3 \mu \mathrm{g} / \mathrm{cm}^{2}\right)$ flasks were used to support the growth of Keratinocytes and SCC12B2 cells. All cells were cultured in a humidified atmosphere with $5 \% \mathrm{CO} 2$ and $21 \% \mathrm{O} 2$ at $37^{\circ} \mathrm{C}$.

\section{Conditioned media}

Human derma fibroblast cell strains FF95, FFRa and FFPia were passaged to reach their replicative senescence at cumulative population doublings (CPDs) $>52,48$ and 42 , respectively, when the typical senescence-associated characteristics [67] appeared. To prepare the conditioned medium $(\mathrm{CM})$, replicative senescent and young $(\mathrm{CPD}<22)$ fibroblasts and intrinsically aged cSCC-associated primary stromal fibroblasts derived from elderly patients were seeded at a density of $1.25 \times 10^{4}$ cells $/ \mathrm{cm}^{2}$ in DMEM growth media. Conditioned media (CM) were harvested from confluent cells after 7 days, centrifuged $(5 \mathrm{~min}$, $400 \times \mathrm{g})$, filtered $(0.22 \mathrm{~mm})$ and normalized to $5 \times 10^{4}$ cells $/ \mathrm{ml}$. Aliquots were stored at $-80^{\circ} \mathrm{C}$ until further use.

\section{Human skin samples}

Human skin specimens were collected from old $(76 \pm 10)$ and young $(21 \pm 8)$ healthy individuals and cSCC patients (Supplementary Table S1) from the Department of Dermatology and Allergic Diseases upon approval by the Ulm University ethical committee (assigned number 155/2012) according to the Declaration of Helsinki principles, after informed written consent was obtained.

\section{Cell migration and invasion assays}

Transwell $^{\circledR}$ migration assay was performed as earlier described [33] with few modifications using Corning ${ }^{\circledR}$ inserts (\#3422, Corning, NY, USA). Recombinant human (rh) Chemerin (\#2324-CM/CF) and rh RANTES/CCL5 (\#278-RN/CF) were purchased from R\&D Systems (Minneapolis, MN, USA). FR180204 (\#SML0320) and SP600125 (\#S5567) were obtained from Sigma-Aldrich (St. Luis, MO, USA), and BIRB796 (\#SM11) from Cell Guidance Systems (St. Luis, MO, USA). Stock solutions were prepared at a concentration of $60 \mathrm{mM}$ in Dimethyl sulfoxide (DMSO).

In brief, a number of $2 \times 10^{5}$ cells in $200 \mu \mathrm{l}$ serumfree DMEM was placed onto the upper chambers. The lower chambers were loaded with $600 \mu \mathrm{l}$ conditioned medium $(\mathrm{CM})$ or chemotaxis factors and incubated overnight $(14 \mathrm{~h})$ at $37^{\circ} \mathrm{C}$ and $5 \% \mathrm{CO} 2$. DMEM containing $10 \%$ fetal bovine serum (FBS) and collagen type I at a 
concentration of $50 \mu \mathrm{g} / \mathrm{ml}$ served as positive chemotaxis controls. DMEM served as a negative control. After 14 hours incubation, cells were fixed and stained using Diff Quik ${ }^{\circledR}$ Stain kit (Medion Diagnostics AG, Switzerland). Non-migrated cells were removed from the upper chambers using cotton swaps. Perforated filters were cut and stuck on slides. Average number of migrated cells were counted in five different high-power microscopic fields (HPF) (×100 magnification).

Invasion assay was performed similar to the migration assay, with a difference that a layer of $\mathrm{BD}$ Matrigel $^{\mathrm{TM}}$ (\#354230, BD Biosciences, USA) was coated $\left(100 \mu \mathrm{l} /\right.$ well) onto the upper filter of Transwell ${ }^{\circledR}$ chambers 4 hours prior to experiment.

\section{Quantitative real-time PCR (qRT-PCR)}

Total RNA was extracted and reverse transcribed using standard protocols. Human Chemokine and Chemokine receptor $\mathrm{RT}^{2}$ Profiler ${ }^{\mathrm{TM}} \mathrm{PCR}$ Array and specific QuantiTect primers (Qiagen, Hilden, Germany,) for RARRES2, CCRL2; GPR1, CMKLR1, CCR1, CCR2, CCR3, CCR4, CCR5, CXCR1 and CXCR2 genes were purchased from Qiagen (Supplementary Table S2) and used according to the manufacturer's instructions.

\section{Immunofluorescence staining}

Immunofluorescence staining was performed using the previously described standard method [68]. Primary antibodies targeting the following proteins were used at indicated concentrations: Chemerin (\#ab72965, 1:50, Abcam, Cambridge, UK), FSP-1 (\#GTX62977, 1:200; GenetTex, Irvine, CA, USA), Cytokeratin (\#M3515, 1:100, Dako, Bollschweil, Germany), CCRL2 (\#ab136057, 1:100, Abcam). Appropriate isotype controls were used to determine the background. The images were acquired using AxioVision software (Carl Zeiss Microimaging GmbH, Jena, Germany).

\section{Western blotting}

Western blot analysis was performed as earlier described [68, 69] using antibodies against CCRL2 (\#ab136057, 1:1000, Abcam), total and phosphorylated MAPK member proteins (Cell Signaling, Massachusetts, USA) including p42/44 (\#4695, 1:2000 \& \#4370, 1:2000), SEK1/MKK4 (\#3346, 1:1000 \& \#4514, 1:1000), ATF-2 (\#9226, 1:1000 \& \#5112, 1:1000), c-Jun (\#9165 \& \#2361, 1:1000) and p38 (\#4511, 1:2000 \& \#8690, 1:2000).

\section{Flow cytometry}

CCRL2, GPR1 and CMKLR1 cell surface expression was measured using APC-conjugated antiCCRL2 (\#FAB23501A, 1:100, R\&D Systems), Alexa488- conjugated anti-GPR1 mAb (\#bs-13509R-A488, 1:100, Bioss, Woburn, MA, USA) and PE-conjugated CMKLR1 (\#FAB362P, 1:100, R\&D systems) according to the manufacturer's recommendations. Appropriate isotype controls were used to determine the background. Dead cells were excluded by co-staining with SYTOX ${ }^{\circledR}$ Blue (Invitrogen, Carlsbad, CA, USA). Flow cytometry was performed on FACSCanto II (BD Biosciences) and the data were analyzed using FlowJo analysis software (TreeStar Inc.).

\section{ELISA}

ELISAs for human Chemerin were performed using Quantikine ELISA kit (R\&D Systems) according to the manufacturer's instructions.

\section{Cignal $^{\mathrm{TM}}$ finder GPCR signaling reporter array}

The GPCR array (\#CCA-109L-2, Qiagen) was used according to manufacturer's protocol. Briefly, SCL-1 cells were seeded at a density of $4 \times 10^{4}$ cells/well and incubated for $18 \mathrm{~h}$ at $5 \% \mathrm{CO}_{2}$ and $37^{\circ} \mathrm{C}$. Following transfection, medium was changed to DMEM containing $40 \mathrm{nM}$ rh Chemerin. After $1 \mathrm{~h}$ (and $24 \mathrm{~h}$ ) incubations, cells were lysed and luciferase expression was determined using Dual-Glo ${ }^{\circledR}$ Luciferase Assay according to manufacturer's instructions (Promega, Madison, WI, USA).

\section{SiRNA-based knockdown of Chemerin}

Fibroblasts $\left(1 \times 10^{5}\right.$ cells/well $)$ were transfected with four different silencing RNAs (siRNAs; $30 \mathrm{nM}$ ) against Chemerin (\# GS5919, Qiagen, Supplementary Table S3) or scrambled control (\#1027280, Qiagen) using Lipofectamine RNAi Max Transfection Reagent (\#13778, Thermo Fisher Scientific, USA). After $42 \mathrm{~h}$ and $48 \mathrm{~h}$ post transfection, quantitative RNA transcripts and secreted Chemerin in the supernatants were analyzed, respectively.

\section{ShRNA-mediated knockdown of CCRL2 and GPR1 in SCL-1}

For the stable knockdown of CCRL2 and GPR1, short hairpin RNA (shRNA) plasmids were obtained from Genecoepia (Rockville, MD, USA, Supplementary Table S4). HEK293T cells $\left(4 \times 10^{6} / 10 \mathrm{~cm}\right.$ dish $)$ were transfected with $13 \mu \mathrm{g}$ shRNA-plasmids along with $8 \mu \mathrm{g}$ psPAX2 and $4 \mu \mathrm{g}$ pMD2 packaging plasmids (Addgene, Cambridge, MA, USA) using TransIT-LTR transfection reagent (Mirus Bio LLC, Madison, WI, USA), and following manufacturer's instructions. Virus was collected 72 hours after transfection, filtered, and stored at $4^{\circ} \mathrm{C}$ for a maximum of one day. The virus titer was assessed and normalized based on the viral protein P24 measurements using a P24 ELISA kit (XpressBio, USA). A number of 
$2 \times 10^{6} \mathrm{SCL}-1$ cells were transduced using equal virus titer. Transduction efficiency was monitored based on eGFP/ mCherry expression, and positively-transduced SCL-1 cells were sorted using FACSAria III (BD Biosciences). Knockdown efficacy was measured using qRT-PCR analysis.

\section{MTT cell viability/proliferation assay}

Cell Proliferation Kit I (MTT) (Roche applied science; Indianapolis, IN) was used for the quantitation of living metabolically active cells as previously described [70]. In brief, SCL- 1 cells $\left(1 \times 10^{4}\right.$ cells/well $)$ were seeded in 96-well plates and serum-starved on the next day. After 24 hours, media was exchanged with DMEM containing rh Chemerin (or DMEM growth media containing MAPK inhibitors) and incubated for 14, 24, 36 and 48 hours.

\section{BrdU proliferation assay}

The effect of Chemerin on the proliferation of SCL-1 was examined by measuring BrdU incorporation into newlysynthesized DNA using immune fluorescence microscopy and flow cytometric analysis as described earlier [70].

\section{Statistics}

The final results were calculated as mean \pm standard error (SEM) of three independent experiments. In some experiments, one of three independent experiment was presented as mean \pm standard deviation (SD). Twotailed unpaired student $t$-test and on-way ANOVA with Bonferroni post hoc test were performed as appropriate, with significance at $p \leq 0.05$ using GraphPad Prism Software Inc (London, UK) unless otherwise stated.

\section{Abbreviations}

ANOVA Analysis of Variance; BrdU 5-bromo2'-deoxyuridine; cSCC Cutaneous squamous cell carcinoma; CCRL2 Chemokine CC-motif receptorlike 2; CM Conditioned medium; CMKLR1 Chemokinelike receptor 1; DMEM Dulbecco's Modified Eagle's Medium; DMSO Dimethyl sulfoxide; ELISA Enzymelinked immunosorbent assay; ERK Extracellular-signalregulated kinase; FBS Fetal bovine serum; GFP Green fluorescent protein; GPCRs G-Protein coupled receptors; GPR1 G-protein coupled receptor 1; HDF Human dermal fibroblast; JNK c-Jun N-terminal kinase; MAPK Mitogen-activated protein kinase; NHEKs Normal human epidermal keratinocytes; qRT-PCR quantitative reverse transcription-polymerase chain reaction; RANTES Regulated on activation, normal $\mathrm{T}$ cell expressed and secreted; RARRES2 Retinoic acid receptor responder 2; SASP Senescence-associated secretory phenotype; SD Standard derivation; SEM Standard error of the Mean; ShRNA Short hairpin RNA; SiRNA Small interfering RNA; TIG2 Tazarotene-induced gene 2

\section{ACKNOWLEDGMENTS AND FUNDING}

We are grateful to Dr. Petra Boukamp (University Hospital Heidelberg), and Dr. James G. Rheinwald (Harvard Medical School) for providing the cSCC cell lines. Primary cSCC-associated fibroblasts were provided by the biobank of the University Research Priority Program (URPP) in translational oncology at the University of Zurich. We thank the technical supports of the Flow Cytometry Core Facilities of the Institute for Experimental Cancer Research and Institute for Physiological Chemistry of Ulm University, Germany. We also gratefully appreciate the technical assistance from Adelheid Hainzl (Department of Dermatology, University of Ulm) and Alice Langer (Department of Dermatology, University Hospital Zurich).

This study is supported by the German Research Foundation (DFG) and a PhD studentship within the International Graduate School in Molecular Medicine, Ulm, Germany.

\section{CONFLICTS OF INTEREST}

The authors declare no conflicts of interest.

\section{REFERENCES}

1. Lomas A, Leonardi-Bee J, Bath-Hextall F. A systematic review of worldwide incidence of nonmelanoma skin cancer. Br J Pharmacol. 2012; 166:1069-1080.

2. Lapouge G, Youssef KK, Vokaer B, Achouri Y, Michaux C, Sotiropoulou PA, Blanpain C. Identifying the cellular origin of squamous skin tumors. Proc Natl Acad Sci. 2011; 108:7431-7436.

3. Lim YZ, South AP. Tumour-stroma crosstalk in the development of squamous cell carcinoma. Int J Biochem Cell Biol. 2014; 53:450-458.

4. Wischermann K, Popp S, Moshir S, Scharfetter-Kochanek K, Wlaschek M, de Gruijl F, Hartschuh W, Greinert R, Volkmer B, Faust A, Rapp A, Schmezer P, Boukamp P. UVA radiation causes DNA strand breaks, chromosomal aberrations and tumorigenic transformation in HaCaT skin keratinocytes. Oncogene. 2008; 27:4269-4280.

5. Kubo Y, Murao K, Matsumoto K, Arase S. Molecular carcinogenesis of squamous cell carcinomas of the skin. J Med Invest. 2002; 49:111-117.

6. Coppé JP, Desprez PY, Krtolica A, Campisi J. The senescence-associated secretory phenotype: the dark side of tumor suppression. Annu Rev Pathol. 2010; 5:99-118.

7. Ohtani N, Takahashi A, Mann DJ, Hara E. Cellular senescence: a double-edged sword in the fight against cancer. Exp Dermatol. 2012; 21:1-4.

8. Ressler S, Bartkova J, Niederegger H, Bartek J, ScharffetterKochanek K, Jansen-Dürr P, Wlaschek M. p16INK4A is a 
robust in vivo biomarker of cellular aging in human skin. Aging Cell. 2006; 5:379-389.

9. Campisi J. Aging, cellular senescence, and cancer. Annual review of physiology. 2013; .

10. Krtolica A, Parrinello S, Lockett S, Desprez PY, Campisi J. Senescent fibroblasts promote epithelial cell growth and tumorigenesis: a link between cancer and aging. Proc Natl Acad Sci. 2001; 98:12072.

11. Golomb L, Sagiv A, Pateras IS, Maly A, Krizhanovsky V, Gorgoulis VG, Oren M, Ben-Yehuda A. Age-associated inflammation connects RAS-induced senescence to stem cell dysfunction and epidermal malignancy. Cell Death Differ. 2015; 22:1764-1774.

12. Davalos AR, Coppe J-P, Campisi J, Desprez P-Y. Senescent cells as a source of inflammatory factors for tumor progression. Cancer Metastasis Rev. 2010; 29:273-283.

13. Singh S, Sadanandam A, Singh RK. Chemokines in tumor angiogenesis and metastasis. Cancer Metastasis Rev. 2007; 26:453-467.

14. Muller A, Homey B, Soto H, Ge N, Catron D, Buchanan ME, McClanahan T, Murphy E, Yuan W, Wagner SN, Barrera JL, Mohar A, Verastegui E, et al. Involvement of chemokine receptors in breast cancer metastasis. Nature. 2001; 410:50-56.

15. Xu Q, Wang Z, Chen X, Duan W, Lei J, Zong L, Li X, Sheng L, Ma J, Han L, Li W, Zhang L, Guo K, et al. Stromalderived factor-1alpha/CXCL12-CXCR4 chemotactic pathway promotes perineural invasion in pancreatic cancer. Oncotarget. 2015; 6:4717-4732. doi: 10.18632/oncotarget.3069.

16. Sarvaiya PJ, Guo D, Ulasov I, Gabikian P, Lesniak MS. Chemokines in tumor progression and metastasis. Oncotarget. 2013; 4:2171-2185. doi: 10.18632/oncotarget.1426.

17. Shields JD, Emmett MS, Dunn DB, Joory KD, Sage LM, Rigby H, Mortimer PS, Orlando A, Levick JR, Bates DO. Chemokine-mediated migration of melanoma cells towards lymphatics - a mechanism contributing to metastasis. Oncogene. 2007; 26:2997-3005. Epub 2006 Nov 2927.

18. Koizumi K, Kozawa Y, Ohashi Y, Nakamura ES, Aozuka Y, Sakurai H, Ichiki K, Doki Y, Misaki T, Saiki I. CCL21 promotes the migration and adhesion of highly lymph node metastatic human non-small cell lung cancer Lu-99 in vitro. Oncol Rep. 2007; 17:1511-1516.

19. Wang J, Xi L, Hunt JL, Gooding W, Whiteside TL, Chen Z, Godfrey TE, Ferris RL. Expression Pattern of Chemokine Receptor 6 (CCR6) and CCR7 in Squamous Cell Carcinoma of the Head and Neck Identifies a Novel Metastatic Phenotype. Cancer Res. 2004; 64:1861-1866.

20. Wang J, Lu Y, Koch AE, Zhang J, Taichman RS. CXCR6 induces prostate cancer progression by the AKT/mammalian target of rapamycin signaling pathway. Cancer Res. 2008; 68:10367-10376. doi: 10310.11158/10008-15472.CAN10308-12780.

21. Xiao G, Wang X, Wang J, Zu L, Cheng G, Hao M, Sun X, Xue Y, Lu J. CXCL16/CXCR6 chemokine signaling mediates breast cancer progression by pERK1/2-dependent mechanisms. Oncotarget. 2015; 6:14165-14178. doi: 10.18632/oncotarget.3690.

22. Yin F, Xu Z, Wang Z, Yao H, Shen Z, Yu F, Tang Y, Fu D, Lin S, Lu G. Elevated chemokine CC-motif receptorlike 2 (CCRL2) promotes cell migration and invasion in glioblastoma. Biochem Biophys Res Commun. 2012.

23. Akram IG, Georges R, Hielscher T, Adwan H, Berger MR. The chemokines CCR1 and CCRL2 have a role in colorectal cancer liver metastasis. Tumour Biol. 2015; 18:18.

24. Zabel BA, Nakae S, Zúñiga L, Kim J-Y, Ohyama T, Alt C, Pan J, Suto H, Soler D, Allen SJ. Mast cell-expressed orphan receptor CCRL2 binds chemerin and is required for optimal induction of IgE-mediated passive cutaneous anaphylaxis. J Exp Med. 2008; 205:2207-2220.

25. Nagpal S, Patel S, Jacobe H, DiSepio D, Ghosn C, Malhotra M, Teng M, Duvic M, Chandraratna RA. Tazaroteneinduced gene 2 (TIG2), a novel retinoid-responsive gene in skin. J Invest Dermatol. 1997; 109.

26. Meder W, Wendland M, Busmann A, Kutzleb C, Spodsberg N, John H, Richter R, Schleuder D, Meyer M, Forssmann W. Characterization of human circulating TIG2 as a ligand for the orphan receptor ChemR23. FEBS Letters. 2003; 555:495-499.

27. Rourke JL, Muruganandan S, Dranse HJ, McMullen NM, Sinal CJ. Gpr1 is an active chemerin receptor influencing glucose homeostasis in obese mice. J Endocrinol. 2014.

28. Wang N, Wang Q-j, Feng Y-y, Shang W, Cai M. Overexpression of chemerin was associated with tumor angiogenesis and poor clinical outcome in squamous cell carcinoma of the oral tongue. Clin Oral Investig. 2013:1-8.

29. Kumar JD, Holmberg C, Kandola S, Steele I, Hegyi P, Tiszlavicz L, Jenkins R, Beynon RJ, Peeney D, Giger OT. Increased Expression of Chemerin in Squamous Esophageal Cancer Myofibroblasts and Role in Recruitment of Mesenchymal Stromal Cells. PLoS One. 2014; 9:e104877.

30. Kumar JD, Kandola S, Tiszlavicz L, Reisz Z, Dockray GJ, Varro A. The role of chemerin and ChemR23 in stimulating the invasion of squamous oesophageal cancer cells. Br J Cancer. 2016; 114:1152-1159. doi: 1110.1038/ bjc.2016.1193. Epub 2016 Apr 1119.

31. Wang C, Wu WK, Liu X, To K-F, Chen GG, Yu J, $\mathrm{Ng}$ EK. Increased serum chemerin level promotes cellular invasiveness in gastric cancer: A clinical and experimental study. Peptides. 2014; 51:131-138.

32. van Zijl F, Krupitza G, Mikulits W. Initial steps of metastasis: Cell invasion and endothelial transmigration. Mutat Res. 2011; 728:23-34.

33. Scharffetter-Kochanek K, Klein CE, Heinen G, Mauch C, Schaefer T, Adelmann-Grill BC, Goerz G, Fusenig NE, Krieg TM, Plewig G. Migration of a Human Keratinocyte Cell Line (HACAT) to Interstitial Collagen Type I Is Mediated by the \&agr; 2 $\beta 1$-Integrin Receptor. J Invest Dermatol. 1992; 98:3-11.

34. Hassona Y, Cirillo N, Heesom K, Parkinson EK, Prime SS. Senescent cancer-associated fibroblasts secrete active 
MMP-2 that promotes keratinocyte dis-cohesion and invasion. Br J Cancer. 2014; 111:1230-1237.

35. Coppé JP, Patil CK, Rodier F, Sun Y, Muñoz DP, Goldstein J, Nelson PS, Desprez PY, Campisi J. Senescence-associated secretory phenotypes reveal cell-nonautonomous functions of oncogenic RAS and the p53 tumor suppressor. PLoS biol. 2008; 6:e301.

36. Coppé J-P, Patil CK, Rodier F, Krtolica A, Beauséjour CM, Parrinello S, Hodgson JG, Chin K, Desprez P-Y, Campisi J. A Human-Like Senescence-Associated Secretory Phenotype Is Conserved in Mouse Cells Dependent on Physiological Oxygen. PLoS One. 2010; 5:e9188.

37. Liu D, Hornsby PJ. Senescent Human Fibroblasts Increase the Early Growth of Xenograft Tumors via Matrix Metalloproteinase Secretion. Cancer Res. 2007; 67:3117-3126.

38. Cahu J, Bustany S, Sola B. Senescence-associated secretory phenotype favors the emergence of cancer stem-like cells. Cell Death Dis. 2012; 20:183.

39. Buettner M, Meyer B, Schreck S, Niedobitek G. Expression of RANTES and MCP-1 in epithelial cells is regulated via LMP1 and CD40. International Journal of Cancer. 2007; 121:2703-2710.

40. Pirilä E, Väyrynen O, Sundquist E, Päkkilä K, Nyberg P, Nurmenniemi S, Pääkkönen V, Pesonen P, Dayan D, Vered M. Macrophages modulate migration and invasion of human tongue squamous cell carcinoma. PLoS One. 2015; 10:e0120895.

41. Giese A, Loo MA, Tran N, Haskett D, Coons SW, Berens ME. Dichotomy of astrocytoma migration and proliferation. Int J Cancer. 1996; 67:275-282.

42. Garay T, Juhász É, Molnár E, Eisenbauer M, Czirók A, Dekan B, László V, Hoda MA, Döme B, Tímár J, Klepetko W, Berger W, Hegedüs B. Cell migration or cytokinesis and proliferation? - Revisiting the "go or grow" hypothesis in cancer cells in vitro. Exp Cell Res. 2013; 319:3094-3103.

43. Ohori M, Kinoshita T, Okubo M, Sato K, Yamazaki A, Arakawa H, Nishimura S, Inamura N, Nakajima $H$, Neya M. Identification of a selective ERK inhibitor and structural determination of the inhibitor-ERK2 complex. Biochem Biophys Res Commun. 2005; 336:357-363.

44. Masamune A, Kikuta K, Suzuki N, Satoh M, Satoh K, Shimosegawa T. A c-Jun NH2-Terminal Kinase Inhibitor SP600125 (Anthra[1,9-cd]pyrazole-6 (2H)-one) Blocks Activation of Pancreatic Stellate Cells. J Pharmacol Exp Ther. 2004; 310:520-527.

45. Kuma Y, Sabio G, Bain J, Shpiro N, Marquez R, Cuenda A. BIRB796 inhibits all p38 MAPK isoforms in vitro and in vivo. J Biol Chem. 2005; 280:19472-19479. Epub 12005 Mar 19478.

46. Bain J, Plater L, Elliott M, Shpiro N, Hastie CJ, McLauchlan H, Klevernic I, Arthur JS, Alessi DR, Cohen P.
The selectivity of protein kinase inhibitors: a further update. Biochem J. 2007; 408:297-315.

47. Demaria M, Desprez PY, Campisi J, Velarde MC. Cell Autonomous and Non-Autonomous Effects of Senescent Cells in the Skin. J Invest Dermatol. 2015; 9:108.

48. Aifuwa I, Giri A, Longe N, Lee SH, An SS, Wirtz D. Senescent stromal cells induce cancer cell migration via inhibition of RhoA/ROCK/myosin-based cell contractility. Oncotarget. 2015; 6:30516-30531. doi: 10.18632/oncotarget.5854.

49. Roh SG, Song SH, Choi KC, Katoh K, Wittamer V, Parmentier M, Sasaki S. Chemerin - a new adipokine that modulates adipogenesis via its own receptor. Biochem Biophys Res Commun. 2007; 362:1013-1018. Epub 2007 Aug 1027.

50. Bondue B, Wittamer V, Parmentier M. Chemerin and its receptors in leukocyte trafficking, inflammation and metabolism. Cytokine Growth Factor Rev. 2011; 22:331-338. doi: 310.1016/j.cytogfr.2011.1011.1004. Epub 2011 Nov 1025.

51. Kaur J, Adya R, Tan BK, Chen J, Randeva HS. Identification of chemerin receptor (ChemR23) in human endothelial cells: chemerin-induced endothelial angiogenesis. Biochem Biophys Res Commun. 2010; 391:1762-1768.

52. Yamaguchi Y, Du XY, Zhao L, Morser J, Leung LL. Proteolytic cleavage of chemerin protein is necessary for activation to the active form, Chem157S, which functions as a signaling molecule in glioblastoma. J Biol Chem. 2011; 286:39510-39519. doi: 39510.31074/jbc.M39111.258921. Epub 252011 Sep 258923.

53. Pachynski RK, Zabel BA, Kohrt HE, Tejeda NM, Monnier J, Swanson CD, Holzer AK, Gentles AJ, Sperinde GV, Edalati A. The chemoattractant chemerin suppresses melanoma by recruiting natural killer cell antitumor defenses. J Exp Med. 2012; 209:1427-1435.

54. Sarmadi P, Tunali G, Esendagli-Yilmaz G, Yilmaz KB, Esendagli G. CRAM-A indicates IFN- $\gamma$-associated inflammatory response in breast cancer. Mol Immunol. 2015; 68:692-698.

55. Hou T, Liang D, Yang D, He J, Huang Y, Zhang Y. High expression of CRAM correlates with poor prognosis in patients with cervical carcinoma. Int J Clin Exp Pathol. 2014; 7:1060-1068. eCollection 2014.

56. Banas M, Zegar A, Kwitniewski M, Zabieglo K, Marczynska J, Kapinska-Mrowiecka M, LaJevic M, Zabel BA, Cichy J. The expression and regulation of chemerin in the epidermis. PLoS One. 2015; 10:e 0117830. doi: 0117810.0111371/journal.pone.0117830. eCollection 0112015.

57. Zheng Y, Luo S, Wang G, Peng Z, Zeng W, Tan S, Xi Y, Fan J. Downregulation of tazarotene induced gene-2 (TIG2) in skin squamous cell carcinoma. Eur J Dermatol. 2008; 18:638-641.

58. Lu X, Kang Y. Chemokine (CC motif) ligand 2 engages CCR2+ stromal cells of monocytic origin to promote breast 
cancer metastasis to lung and bone. J Biol Chem. 2009; 284:29087.

59. Hart R, Greaves DR. Chemerin Contributes to Inflammation by Promoting Macrophage Adhesion to VCAM-1 and Fibronectin through Clustering of VLA-4 and VLA-5. J Immunol. 2010; 185:3728-3739.

60. Dhillon AS, Hagan S, Rath O, Kolch W. MAP kinase signalling pathways in cancer. Oncogene. 2007; 26:3279-3290.

61. Mikuła-Pietrasik J, Sosińska P, Maksin K, Kucińska M, Piotrowska H, Murias M, Woźniak A, Szpurek D, Książek K. Colorectal cancer-promoting activity of the senescent peritoneal mesothelium. Oncotarget. 2015; 6:29178-29195. doi: 10.18632/oncotarget.4932.

62. Kaur A, Webster MR, Marchbank K, Behera R, Ndoye A, Kugel CH, 3rd, Dang VM, Appleton J, O'Connell MP, Cheng P, Valiga AA, Morissette R, et al. sFRP2 in the aged microenvironment drives melanoma metastasis and therapy resistance. Nature. 2016; 532:250-254.

63. Rheinwald JG, Beckett MA. Tumorigenic keratinocyte lines requiring anchorage and fibroblast support cultured from human squamous cell carcinomas. Cancer Res. 1981; 41:1657-1663.

64. Tilgen W, Boukamp P, Breitkreutz D, Dzarlieva R, Engstner M, Haag D, Fusenig N. Preservation of Morphological, Functional, and Karyotypic Traits during Long-Term Culture and in Vivo Passage of Two Human Skin Squamous Cell Carcinomas. Cancer Res. 1983; 43:5995-6011.

65. Fleischmajer R, Perlish JS, Krieg T, Timpl R. Variability in Collagen and Fibronectin Synthesis by Scleroderma Fibroblasts in Primary Culture. J Invest Dermatol. 1981; 76:400-403.
66. Naderi-Hachtroudi L, Peters T, Brenneisen P, Meewes C, Hommel C, Razi-Wolf Z, Schneider LA, Schuller J, Wlaschek M, Scharffetter-Kochanek K. Induction of manganese superoxide dismutase in human dermal fibroblasts: a UV-B-mediated paracrine mechanism with the release of epidermal interleukin 1 alpha, interleukin 1 beta, and tumor necrosis factor alpha. Arch Dermatol. 2002; 138:1473-1479.

67. Rodier F, Coppe J-P, Patil CK, Hoeijmakers WAM, Munoz DP, Raza SR, Freund A, Campeau E, Davalos AR, Campisi J. Persistent DNA damage signalling triggers senescence-associated inflammatory cytokine secretion. Nat Cell Biol. 2009; 11:973-979.

68. Jiang D, Qi Y, Walker NG, Sindrilaru A, Hainzl A, Wlaschek M, MacNeil S, Scharffetter-Kochanek K. The effect of adipose tissue derived MSCs delivered by a chemically defined carrier on full-thickness cutaneous wound healing. Biomaterials. 2013; 34:2501-2515.

69. Treiber N, Maity P, Singh K, Kohn M, Keist AF, Ferchiu F, Sante L, Frese S, Bloch W, Kreppel F. Accelerated aging phenotype in mice with conditional deficiency for mitochondrial superoxide dismutase in the connective tissue. Aging Cell. 2011; 10:239-254.

70. Singh K, Maity P, Krug L, Meyer P, Treiber N, Lucas T, Basu A, Kochanek S, Wlaschek M, Geiger H, ScharffetterKochanek K. Superoxide anion radicals induce IGF-1 resistance through concomitant activation of PTP1B and PTEN. EMBO Mol Med. 2014; 7:59-77. doi: 10.15252/ emmm.201404082. 